\title{
A bridge to progress further afield: The promise of a common framework on attentional capture
}

\section{Steven B. Most}

School of Psychology, UNSW Sydney, Sydney NSW 2052, Australia

email: s.most@unsw.edu.au

\section{Kim M. Curby}

Department of Psychology, Macquarie University, Sydney NSW 2109, Australia; Centre for Elite Performance, Expertise, \& Training, Macquarie University, Sydney, Australia email: kim.curby@mq.edu.au

\begin{abstract}
Although physical salience looms large in the attentional capture literature, stimuli can also capture attention via salience deriving from non-physical factors. Such psychological salience can stem, for example, from the emotional resonance of stimuli or their relevance to a person's expertise. We consider how insights from a recently proposed framework for attentional capture can be used to advance theory and drive research on the role of emotion-driven attentional biases in clinical disorders and on how attentional allocation changes with the development of perceptual expertise. In return, we wonder how their common framework can be enriched through considerations of psychological salience.
\end{abstract}

The coming together of traditionally adversarial perspectives on attentional capture in service of a common theoretical framework by Luck, Gaspelin, Folk, Remington, and Theeuwes (2021; i.e., the target article) is a milestone in this decades-old literature. Beyond the benefits for the attentional capture literature itself, the resulting framework can and should advance theory in other parts of the field. In this brief commentary, we highlight examples of how it might do so.

In particular, although the current framework applies to how physical salience combines with selection history and attentional control settings to determine attentional capture, there is much that can be applied to (or in some cases, perhaps, honed by) understanding of how psychologically salient stimuli capture attention. Indeed, evidence suggests that stimuli can 
capture attention because of their relation to what the perceiver brings to the visual scene, either because of (for example) years of developed expertise or their emotions. In some cases, such as in the case of emotion-driven capture, the literatures on these topics echo distinctions drawn within the visual cognitive approach (represented in the current target article), and in these cases the labor of building bridges between researchers begins with translating the concepts and terminology of one literature into the other's. In other cases, such as the impact of perceptual expertise on attentional allocation, the framework described in the target article introduces potentially new ways of thinking about existing topics of investigation and debate. In return, findings from the clinical, emotion, and expertise literatures may help point the way towards incorporating psychological salience into an even more unified framework.

Attention and emotion. The clinical literature on attentional biases and their role in a range of psychological disorders may be a candidate for conceptual overlap, where advances can be facilitated by explicitly mapping existing concepts onto Luck and colleagues' proposed framework. Benefits would come, however, not from merely swapping terms between literatures. Rather, the framework proposed in the target article provides a mechanistic architecture, based on leading-edge research and theory on attention, that can recalibrate understanding of how emotion and attention influence each other, of the probable loci of individual differences, and of information processing mechanisms that may serve as promising targets for intervention.

Emotional stimuli are known to capture attention, a pattern that is particularly robust in clinical populations. For example, threat-related stimuli tend to capture attention among anxious individuals (e.g., MacLeod, Mathews, \& Tata, 1986), trauma-related stimuli capture attention among individuals with PTSD (e.g., Olatunji et al., 2013), and food images capture attention among individuals with anorexia nervosa (Neimeijer et al, 2017). Such attentional biases can be so characteristic of a range of disorders that they are sometimes thought to play a causal or exacerbating role. One prominent hypothesis is that anxiety-prone individuals are hypervigilant to signs of threat in the environment, a state that feeds into a cycle that culminates in severe, clinical anxiety because the inevitably more frequent detection of perceived threats serves to validate anticipation of danger (e.g., Eysenck, 1992). On the face of it, this hypervigilance may map onto the concept of "attentional set" in the visual cognition literature, though in this case it would be an attentional set that is chronic, sustained, and implicit. 
Concepts core to the target article's framework, such as priority signals, signal suppression, and reactive and proactive control, seem to provide mechanistic scaffolding for parallel notions found in information processing approaches to anxiety. For example, patterns of response time in spatial attention tasks such as spatial cueing and the classic "dot probe" have been used to assess the time-courses of attentional orienting to and disengagement from threatening stimuli (e.g., Fox et al., 2001). These, in turn, have been used to support models suggesting that the threat values of stimuli are assessed "pre-attentively", with either the perceived threat level or trait anxiety determining whether attention is reflexively allocated towards or away from them (e.g., Williams et al., 1997). Research testing these models can gain much by recasting them in terms of the target article's framework. Notions of preattentive threat evaluation and individual differences in attentional allocation may more usefully be framed as testable questions of whether threat evaluations manifest as enhanced priority signals (e.g., Notebaert et al., 2011) or instead come into play, as the presence or absence of signal suppression, only after saliency computations have operated upon physical features. Such reframing would also yield additional opportunities to build bridges with the emotion literature; for example, to the degree that emotion contributes to salience computations, what aspects of emotion play this role: emotional valence (positive vs. negative) or emotional arousal, which are two primary dimensions along which emotions vary?

If it turns out that emotion does not modulate saliency computations themselves, the framework's consideration of the nature of a control state can also be applied towards understanding of attentional capture by emotional stimuli. To a degree, such distinctions have already been applied, as questions of proactive and reactive control over emotional distraction have been raised. In one investigation, proactive control eliminated emotional distraction, whereas reactive control did not (Grimshaw et al., 2018). In that case, proactive control was instantiated through manipulations of distractor frequency, consistent with notions (found in the framework by Luck and colleagues) that implicit learning can mitigate attentional capture. An open question is whether implicit learning serves as a more effective mitigator of emotional distraction than explicit control settings.

Recasting the literature on emotion-driven attentional biases in light of contemporary understanding of attention mechanisms has a practical purpose in addition to its potential to enrich theory. Notions that attentional biases can play a causal or exacerbating role in a range 
of disorders has led to explorations of "attentional bias modification" (ABM) as a form of intervention (see Bar-Haim et al., 2007). Yet, evidence for the effectiveness of ABM is mixed. One possibility is that efforts to retrain attentional biases have targeted the wrong attention mechanisms; most often, $\mathrm{ABM}$ takes the form of a spatial dot probe task in which people train to orient away from threatening stimuli, but a finer-grain delineation of attention mechanisms can allow more targeted training. It's a fair question whether such training can be effective even when the optimal attention mechanisms are targeted, but in our own work we have found that frequent players of violent video games are less distracted by graphic emotional images (Jin et al., 2018). Could this be because years of exposure to violent imagery decreased the priority signal of such images? Alternatively, the locus of this effect may have involved reactive or proactive control mechanisms (leaving aside the possibility that this link was correlational and not causal). Although a common assumption in the clinical literature is that various measures of attentional bias generally tap into the same underlying construct (see Van Bockstaele et al., 2014, p. 688), the framework proposed by Luck and colleagues not only shows that attention is multi-faceted, but also provides an organized taxonomy of attention mechanisms that may be more or less linked with clinically relevant individual differences.

Attention and expertise. Real-world experience, especially in the case of perceptual experts, provides another example of a factor that can impact the way people reflexively direct attention independent of physical salience. Although effects of perceptual expertise are not typically framed in ways that correspond closely to concepts within Luck and colleagues' framework, they may be a case where this framework can inspire new ways of conceptualizing old questions. As people become visual experts with a stimulus class, their perception of these objects becomes more "holistic", with evidence suggesting this is largely due to changes in attentional allocation (Chua, Richler, \& Gauthier, 2015). Strikingly, these changes can lead experts to perform worse on some tasks than novices: when people are tasked with making judgments about part of an object-of-expertise (e.g., the bottom part in a "part-matching" task), it is experts who have the most trouble avoiding interference from the non-target part (e.g., Gauthier et al, 2003). Such patterns have been described as a failure of selective attention to parts, with attention reflexively allocated to both regions of the stimulus. Consistent with this, manipulations that impede the spread of attention from the target half to the non-target half reduce this interference (e.g., Curby, Entenman, \& Fleming, 2016). 
While there is growing evidence that holistic processing is a result of a learned pattern of attention to an expert category (Chua, Richler, \& Gauthier, 2015), little is known about the mechanisms supporting this change in attentional allocation. One possibility is that, in the context of part-matching tasks, the task-irrelevant parts (which are otherwise relevant to experts in most contexts) capture experts' attention. The framework in the target article largely discusses attentional capture by discrete stimuli, and it is less clear whether it can be applied as well to attentional capture by sub-elements within objects. A study in which lung nodules within a chest radiograph captured attention among trained radiologists suggests that diagnostic sub-elements of a stimulus can capture attention (Carrigan et al., 2019). If the framework can be so applied, it provides a potentially attractive path forward for the expertise literature. For example, researchers can ask whether holistic processing emerges because learning leads to a more distributed "attend to me" signal across the object of expertise. Do the non-target parts grab attention among experts because years of training have imbued these visual elements with an enhanced priority signal? Would this occur simply because of selection history, learned attentional set, or because of some other contributing factor to perceptual expertise? Viewing the perceptual expertise literature through the lens of the target article's framework opens new opportunities for refining theoretical understanding of how attention and perception change with experience.

Conclusion. Attentional capture is central to a wide range of research topics and theories in psychology, many of which are principally concerned with the implications and downstream consequences of attentional capture by psychological salience. Researchers in these areas rarely train their metaphorical microscopes on fine-grained distinctions between mechanisms of attention, so building bridges that connect these areas to insights from the visual cognitive approach can be a powerful way both to refine understanding of attentional capture in a broad range of contexts and to enhance the utility and impact of lab-derived mechanistic frameworks. For researchers from areas like clinical psychology or the study of higher-level skilled performance, who may have enthusiastically greeted such opportunities, such bridges may have been difficult to build until now because competing perspectives within the visual cognition literature had not established common ground suitable enough to build on. The milestone achievement by Luck, Gaspelin, Folk, Remington, and Theeuwes (2021), in the current target article, therefore provides an exciting opportunity both for research on attentional capture itself and for research more broadly. In this commentary, we highlighted two literatures - on emotion-driven attentional biases and perceptual expertise - where the 
target article's framework can help advance research and theory. We suggest, also, that these literatures (and potentially others) may in return offer opportunities to gain new insights into attentional capture. As research progresses, it will be important to understand how psychological salience - in addition to physical salience - plays a role in this new common framework.

\section{Acknowledgement}

Thank you to Lies Notebaert for helpful comments on this commentary.

\section{References}

Bar-Haim, Y., Lamy, D., Pergamin, L., Bakermans-Kranenburg, M. J., \& van IJzendoorn, M. H. (2007). Threat-related attentional bias in anxious and nonanxious individuals: a metaanalytic study. Psychological bulletin, 133(1), 1-24. https://doi.org/10.1037/00332909.133.1.1

Carrigan, A. J., Curby, K. M., Moerel, D., \& Rich, A. N. (2019). Exploring the effect of context and expertise on attention: is attention shifted by information in medical images? Attention, perception \& psychophysics, 81(5), 1283-1296. https://doi.org/10.3758/s13414019-01695-7

Chua, K. W., Richler, J. J., \& Gauthier, I. (2015). Holistic processing from learned attention to parts. Journal of experimental psychology. General, 144(4), 723-729. https://doi.org/10.1037/xge0000063

Curby, K. M., Entenman, R. J., \& Fleming, J. T. (2016). Holistic face perception is modulated by experience-dependent perceptual grouping. Attention, perception \& psychophysics, 78(5), 1392-1404. https://doi.org/10.3758/s13414-016-1077-8

Eysenck, M. W. (1992). Anxiety: The cognitive perspective. Lawrence Erlbaum Associates, Inc.

Fox, E., Russo, R., Bowles, R., \& Dutton, K. (2001). Do threatening stimuli draw or hold visual attention in subclinical anxiety? Journal of experimental psychology. General, 130(4), 681-700.

Gauthier, I., Curran, T., Curby, K. M., \& Collins, D. (2003). Perceptual interference supports a non-modular account of face processing. Nature neuroscience, 6(4), 428-432. https://doi.org/10.1038/nn1029

Grimshaw, G. M., Kranz, L. S., Carmel, D., Moody, R. E., \& Devue, C. (2018). Contrasting reactive and proactive control of emotional distraction. Emotion (Washington, D.C.), 18(1), 26-38. https://doi.org/10.1037/emo0000337

Jin, M., Onie, S., Curby, K. M., \& Most, S. B. (2018). Aversive images cause less perceptual interference among violent video game players: Evidence from emotion-induced 
blindness. Visual Cognition, 26(10), 753-763.

https://doi.org/10.1080/13506285.2018.1553223

Luck, S. J., Gaspelin, N., Folk, C. L., Remington, R. W., \& Theeuwes, J. (2021). Progress toward resolving the attentional capture debate. Visual Cognition, 29(1), 1-21.

MacLeod, C., Mathews, A., \& Tata, P. (1986). Attentional bias in emotional disorders. Journal of abnormal psychology, 95(1), 15-20. https://doi.org/10.1037//0021$\underline{843 x .95 .1 .15}$

Neimeijer, R., Roefs, A., \& de Jong, P. J. (2017). Heightened attentional capture by visual food stimuli in anorexia nervosa. Journal of Abnormal Psychology, 126(6), 805-811. https://doi.org/10.1037/abn0000275

Notebaert, L., Crombez, G., Van Damme, S., De Houwer, J., \& Theeuwes, J. (2011). Signals of threat do not capture, but prioritize, attention: A conditioning approach. Emotion, 11(1), 81-89. https://doi.org/10.1037/a0021286

Olatunji, B. O., Armstrong, T., McHugo, M., \& Zald, D. H. (2013). Heightened attentional capture by threat in veterans with PTSD. Journal of abnormal psychology, 122(2), 397405. https://doi.org/10.1037/a0030440

Van Bockstaele, B., Verschuere, B., Tibboel, H., De Houwer, J., Crombez, G., \& Koster, E. H. (2014). A review of current evidence for the causal impact of attentional bias on fear and anxiety. Psychological Bulletin, 140(3), 682-721. https://doi.org/10.1037/a0034834

Williams, J. M. G., Watts, F. N., Macleod, C., \& Mathews, A. (1997). Cognitive Psychology and Emotional Disorders. (2 ed.) John Wiley \& Sons. 